\title{
Structural Basis for L230 Enzymatic Regulation in Mimivirus Collagen Biosynthesis
}

\author{
Alvarado $\mathrm{SK}^{1}$, Guo $\mathrm{HF}^{2}$, Kurie $\mathrm{JM}^{2}$, Phillips GN Jr. ${ }^{1}$ \\ 1. Department of Biosciences, Rice University, Houston TX, 77005 \\ 2. Department of Thoracic/Head and Neck Medical Oncology, University of Texas MD \\ Anderson Cancer Center, Houston TX, 77030
}

L230 is a collagen-modifying protein that is isolated from the Acanthamoeba polyphaga mimivirus. It is a multi-domain bifunctional enzyme that is responsible for sequential lysine hydroxylation and glucosylation of mimivirus collagens that are located on the viral capsid. The $2.0 \AA$ apo structure of the truncated hydroxylase domain possesses a double stranded $\beta$-helix fold consisting of eight consecutive $\beta$-strands. L230 is a member of the 2-oxoglutarate dioxygenase superfamily and utilizes a conserved catalytic mechanism for hydroxylation of collagenous lysines. The hydroxylation mechanism requires several cofactors and cosubstrates including 2oxoglutarate, dioxygen and ferrous iron. A highly conserved facial triad consisting of histidine 825 , histidine 877 and aspartic acid 827 are responsible for the coordination of ferrous iron. The atomic structure reveals a distinctive dimeric interface that facilitates the spatial positioning of collagen prior to catalysis. Analysis of the surface architecture displays an extensive cavity that enables sequential diffusion of 2-oxoglutarate and the lysine selected for catalysis. Holo L230 with chemically diverse collagen mimetic peptides will provide structural insights into residues that confer substrate specificity. The atomic L230 hydroxylase domain structural determination provides an introduction into collagen hydroxylation and complements pursuit of L230 whole enzyme structural investigations for insight into bifunctional enzymatic coordination of mimivirus collagen biosynthesis.

This work is supported by a training fellowship from the Gulf Coast Consortia, on the Houston Area Molecular Biophysics Program T32GM008280 and CPRIT CA105155-12. 